\title{
On Lengths of Rainbow Cycles
}

\author{
Boris Alexeev* \\ Department of Mathematics \\ Massachusetts Institute of Technology \\ Cambridge, MA 02139, USA \\ borisa@mit.edu
}

Submitted: Aug 18, 2006; Accepted: Oct 27, 2006; Published: Nov 17, 2006

Mathematics Subject Classifications: 05C15, 05C38

\begin{abstract}
We prove several results regarding edge-colored complete graphs and rainbow cycles, cycles with no color appearing on more than one edge. We settle a question posed by Ball, Pultr and Vojtěchovský by showing that if such a coloring does not contain a rainbow cycle of length $n$, where $n$ is odd, then it also does not contain a rainbow cycle of length $m$ for all $m$ greater than $2 n^{2}$. In addition, we present two examples which demonstrate that a similar result does not hold for even $n$. Finally, we state several open problems in the area.
\end{abstract}

\section{Introduction}

A rainbow cycle within an edge-colored graph is a cycle all of whose edges are colored with distinct colors. Rainbow cycles, sometimes called colorful or totally multicolored cycles in other sources, have been introduced in many different contexts. For example, Burr, Erdős, Sós, Frankl, and Graham [BEGS89, BES ${ }^{+}$91] studied which graphs may be $r$-colored (for a fixed integer $r$ ) so that each copy of some subgraph, in particular a cycle of a certain length, is rainbow.

From another perspective, Erdős, Simonovits, and Sós [ESS75] investigated a function $f\left(n, C_{k}\right)$, defined as the maximum number of colors in which the edges of the complete graph $K_{n}$ on $n$ vertices may be colored so that the coloring contains no rainbow $k$-cycles; they conjectured that $f\left(n, C_{k}\right)=n \cdot((k-2) / 2+1 /(k-1))+O(1)$ for $n \geq k \geq 3$ and proved the case $k=3$. Alon Alo83 proved the conjecture for $k=4$ and derived an upper bound for general $k$; Jiang and West [JW03] further improved these bounds and mentioned that the conjecture has been proven for all $k \leq 7$. Finally, Montellano-Ballesteros and Neumann-Lara MBNL05] recently proved the conjecture completely. More research has

${ }^{*}$ This research is supported by MIT's Paul E. Gray Endowed Fund for UROP.

THE ELECTRONiC JOURNAl OF COMBINATORICS 13 (2006), \#R105 
occurred in related areas; these references are not intended as a comprehensive survey of the area, but rather only as a small sample.

Within this paper, we build on the research of Ball, Pultr, and Vojtěchovský [BPV], who studied rainbow cycles within edge-colored complete graphs. In particular, they asked when the existence of a rainbow cycle of a certain length forces the existence of a rainbow cycle of another length, approaching the problem with applications for distributive lattices. To begin, it is easy to see that within such a coloring, the absence of rainbow $n$-cycles and rainbow $m$-cycles implies the absence of rainbow $(n+m-2)$-cycles. (For example, by this fact alone, the absence of rainbow 3-cycles implies there are no rainbow cycles at all, while the absence of rainbow 4-cycles implies there are no even-length rainbow cycles. In general, if there are no rainbow $n$-cycles, then there are no cycles with lengths congruent to $2 \bmod (n-2)$.) Ball et al. asked whether or not the restrictions implied by this observation were the only restrictions on the lengths of rainbow cycles in a coloring.

We answer this question in the negative by showing that there are more, and much stronger, restrictions on the possible lengths. In particular, our main result states that if a coloring does not contain a rainbow cycle of length $n$, where $n$ is odd, then it also does not contain a rainbow cycle of length $m$ for all $m \geq 2 n^{2}$. These results follow from the observation above combined with intermediate results that show that the absence of a rainbow $n$-cycle (again, with $n$ odd) implies the absence of rainbow cycles of lengths $\left(\begin{array}{l}n \\ 2\end{array}\right)$ and $3 n-6$.

However, the absence of a rainbow cycle of even length does not put the same restrictions on the lengths of longer rainbow cycles. For example, Ball et al. showed that there are colorings with no even-length rainbow cycles, but with rainbow cycles of all odd lengths up to the number of vertices in the graph. We present these colorings, in addition to original colorings in which there are rainbow cycles of all lengths except those congruent to $2 \bmod 4$.

We briefly describe the organization of the paper. We begin with preliminaries, including definitions and the proof of the observation above, allowing us to formulate the original question and the main result formally. In order to provide a flavor of colorings that avoid rainbow cycles, we present the "even-length" examples next. We then prove that the absence of a rainbow $n$-cycle (for $n$ odd) implies the absence of rainbow $\left(\begin{array}{l}n \\ 2\end{array}\right)$ cycles, and show how this prohibits all sufficiently large cycles (with lengths on the order of a cubic in $n$ ). Next, we show the stronger result that the absence of a rainbow $n$-cycle (for $n$ odd) also prohibits rainbow $(3 n-6)$-cycles, implying the absence of all sufficiently large cycles (with lengths on the order of a quadratic in $n$, in particular $2 n^{2}$, the main result). At the end of the paper, we state some still-open problems in the area and present some computer-obtained results.

\section{Preliminaries}

We begin by introducing some conventions and definitions. In this, we mostly follow the earlier work of Ball et al. [BPV], where they introduced the following definitions, as well 
as proved Lemma 1 (and its corollary) and Claim 6.

Definition. In the context of this paper, a coloring is an edge-coloring of an undirected complete graph; the colors may come from an arbitrary set and there is no restriction that the coloring be proper. As above, a rainbow n-cycle (again, sometimes called colorful or totally multicolored in other sources) within a coloring is a cycle consisting of $n$ distinct vertices, all of whose edges are colored with distinct colors; in this case, we will also say that the coloring contains a rainbow $n$-cycle (that is, the coloring restricted to the edges of the cycle is a bijection). As notation, we write $\left(v_{1}, \ldots, v_{n}\right)$ for the cycle that visits vertices $v_{1}, \ldots, v_{n}$ in order (and then returns to $v_{1}$ ). Notice that although we allow infinite graphs, all cycles are of course finite.

A simple lemma and an immediate corollary guide us in our study of rainbow cycles.

Lemma 1. If a coloring contains no rainbow $n$-cycles nor rainbow $m$-cycles, then it contains no rainbow $(n+m-2)$-cycles.

Corollary 2. In particular, if a coloring contains no rainbow n-cycle, then it contains no rainbow cycles of length $\ell$, where $\ell \equiv 2(\bmod n-2)$.

Proof. The proof is by contradiction: assume that the coloring contains a rainbow $(n+$ $m-2$ )-cycle. It can be divided, by a single chord, into an $n$-cycle and an $m$-cycle (see Figure 11). Consider the color of this chord. On the one hand, it must agree with one of the other edges of the $n$-cycle to avoid a rainbow $n$-cycle; on the other hand, it must similarly agree with one of the other edges of the $m$-cycle to avoid a rainbow $m$-cycle. This, however, is a contradiction, as we assumed the outer $(n+m-2)$-cycle was rainbow.

If an $n$-cycle is prohibited, then so are $(2 n-2)$-cycles. By induction, we obtain precisely all lengths congruent to $2 \bmod n-2$.

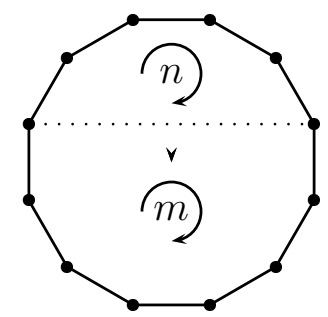

Figure 1: An $(n+m-2)$-cycle divided into an $n$-cycle and an $m$-cycle by a chord. (See Lemma 1].)

\section{Original Question and the Main Result}

The original question [BPV] can be stated succinctly as follows: 
Question 3. Is the restriction in Lemma 1 the only restriction on what lengths of rainbow cycles a coloring can contain? In other words, can any set of lengths that does not contradict the lemma be obtained as the lengths of rainbow cycles of some coloring?

Remark. As we shall see later in the proof of Lemma 10, this question can be stated formally as follows: Is it true that for any submonoid $S \subset \mathbb{N}$ the set of lengths $\{s+2 \mid s \notin$ $S\}$, may be obtained as the lengths of rainbow cycles of some coloring? Note also that if one only considers finite graphs, then it is necessary to add in a restriction prohibiting sets containing arbitrarily large cycle lengths.

We may also formulate a weaker question based on Corollary 2

Question 4. If $m \neq \equiv 2(\bmod n-2)$, does there exist a coloring of $K_{m}$ with a rainbow (Hamiltonian) $m$-cycle but no rainbow $n$-cycle?

However, both questions can be answered in the negative; indeed, they both contradict our main theorem, which is somewhat of an opposite, Ramsey-type result. We state it here but prove it later.

Theorem 5. Suppose $n$ is an odd integer. If a coloring does not contain a rainbow ncycle, it also does not contain a rainbow m-cycle for all sufficiently large $m$; in particular, $m \geq 2 n^{2}$ suffices.

In particular, we shall show that if there is no rainbow 5-cycle, there is also no rainbow 10-cycle, answering Question 4 in the negative with $m=10$ and $n=5$.

\section{The Even Case}

Before proving our result, it is instructive to consider examples of colorings which contain some lengths of rainbow cycles, but yet do not contain many other lengths. In particular, we will construct colorings that show that Theorem $[5$ is not true for even $n$. Of the following two results, Claim [6] was proven in [BPV] but Claim [7] is original.

Claim 6. There exists a coloring col of an infinite complete graph that contains rainbow cycles of all odd lengths, but no even-length rainbow cycles. Furthermore, for each n, taking appropriate finite subgraphs (and their induced colorings) yields colorings that contain rainbow cycles of all odd lengths up to $n$, but still no even-length rainbow cycles.

Proof. We construct col first. Let the vertex set be the positive integers $\mathbb{Z}^{+}$and the colors the nonnegative integers $\mathbb{N}$ and define the color of the edge joining distinct vertices $x$ and $y$ to be

$$
\operatorname{col}(x, y)= \begin{cases}0 & \text { if } y-x \text { is even, } \\ \min (x, y) & \text { if } y-x \text { is odd }\end{cases}
$$

First, we must show that there exist rainbow cycles of all odd lengths. But this is easy! Consider the cycle $(1,2,3, \ldots, k)$ for $k$ odd. For $1 \leq i<k$, the color of the edge joining $i$ 
and $i+1$ is $\operatorname{col}(i, i+1)=i$; finally, the color of the edge joining $k$ and 1 is $\operatorname{col}(k, 1)=0$. These are all distinct, so it remains to show that there are no even-length cycles.

However, by Corollary 2, we need only show that there are no rainbow 4-cycles. Suppose, by contradiction, that there is a rainbow cycle $(a, b, c, d)$. How many times is the case "col $(x, y)=0$ if $y-x$ is even" used along the edges? It cannot be used more than once because otherwise the cycle would contain a repeated color, 0 . But $(b-a)+(c-b)+(d-c)+(a-d)=0$ and thus, by parity, an even number of $b-a, c-b$, $d-c$, and $a-d$ are odd and this case cannot be used exactly once. Therefore, all of the edges use the "col $(x, y)=\min (x, y)$ if $y-x$ is odd" case of the above definition. Now, without loss of generality, assume $a$ is the smallest-numbered vertex of the four. A contradiction is immediate: since $a$ is the smallest-numbered vertex, $\operatorname{col}(a, b)=\operatorname{col}(a, d)=a$. Therefore there are no rainbow 4-cycles and thus no even-length rainbow cycles at all.

Finally, taking the induced subgraph on the vertices from 1 to $n$ accomplishes the second statement of the claim. Indeed, all of the necessary odd-length rainbow cycles mentioned above still exist, and a rainbow 4-cycle still doesn't exist.

Claim 7. There exists another similar coloring $\mathrm{col}^{\prime}$ of an infinite complete graph that contains rainbow cycles of all lengths, except those lengths congruent to 2 mod 4. Furthermore, taking appropriate finite subgraphs has the same effect as before.

Proof. Again, let the vertex set be the positive integers $\mathbb{Z}^{+}$and the colors the nonnegative integers $\mathbb{N}$; then for distinct vertices $x$ and $y$, define

$$
\operatorname{col}^{\prime}(x, y)=\left\{\begin{array}{lll}
0 & \text { if } y-x \equiv 0 & (\bmod 2) \\
x & \text { if } y-x \equiv 1 & (\bmod 4) \\
y & \text { if } y-x \equiv-1 & (\bmod 4)
\end{array}\right.
$$

Note that $\operatorname{col}^{\prime}$ is well-defined, as $\operatorname{col}^{\prime}(x, y)=\operatorname{col}^{\prime}(y, x)$ for all $x$ and $y$. Again, demonstrating the existence of rainbow $k$-cycles, for all $k \neq \equiv 2(\bmod 4)$, is simple; the cycle $(1,2,3, \ldots, k)$ accomplishes the task. For $1 \leq i<k$, the color of the edge joining $i$ and $i+1$ is $\operatorname{col}^{\prime}(i, i+1)=i$; we must only analyze the color of the edge joining $k$ and 1 . If $k$ is odd, then $\operatorname{col}^{\prime}(k, 1)=0$; if $k$ is divisible by 4 , the $\operatorname{color}^{\operatorname{col}^{\prime}}(k, 1)=k$. In either case, the cycle is rainbow.

It remains to show that there is no 6-cycle (once again, Corollary 2 implies we need only check this length). We proceed by contradiction: assume there is a rainbow cycle $(a, b, c, d, e, f)$. As before, the rule " $\operatorname{col}^{\prime}(x, y)=0$ if $y-x \equiv 0(\bmod 2)$ " cannot be used at all. We may also assume $b-a \equiv-1(\bmod 4)$ : if it is $1 \bmod 4$, simply change the direction of the cycle $(a, b, c, d, e, f) \mapsto(b, a, f, e, d, c)$. From this assumption, it follows that $c-b \equiv-1$; indeed, if $c-b \equiv 1(\bmod 4)$, then $\operatorname{col}(a, b)=b=\operatorname{col}(b, c)$. Similarly, we may conclude that $b-a \equiv c-b \equiv d-c \equiv \cdots \equiv a-f \equiv-1(\bmod 4)$. This is a contradiction; clearly $(b-a)+(c-b)+\cdots+(a-f)=0$, but the previous congruence would imply it were equal to $2 \bmod 4$.

Of course, taking the corresponding induced subgraphs achieves the finite results. 


\section{Building up to the Main Result}

We now prove a theorem slightly weaker than the main result by building on two lemmas.

Lemma 8. If a coloring does not contain a rainbow $n$-cycle, where $n=2 k+1$ is odd, it also does not contain a rainbow $m$-cycle, where $m=\left(\begin{array}{l}n \\ 2\end{array}\right)=k \cdot(2 k+1)$.

Remark. The $k=2$ case yields the result involving 5 and 10 mentioned in Section [3,

Proof. We prove the contrapositive. Assume that we have a coloring, col, of $K_{m}$ such that there is a rainbow (Hamiltonian) $m$-cycle but no rainbow $n$-cycle. Without loss of generality, we may number the vertices of the $K_{m}$ by residues modulo $m$ and insist that $\operatorname{col}(i, i+1)=i$ for $i \in \mathbb{Z} / m \mathbb{Z}$; in other words, we assume that our rainbow $m$-cycle is given by $(0,1, \ldots, m-1)$. Consider the following $k+1$ different $(2 k+1)$-cycles: (see Figure 2 for a visual accompaniment)

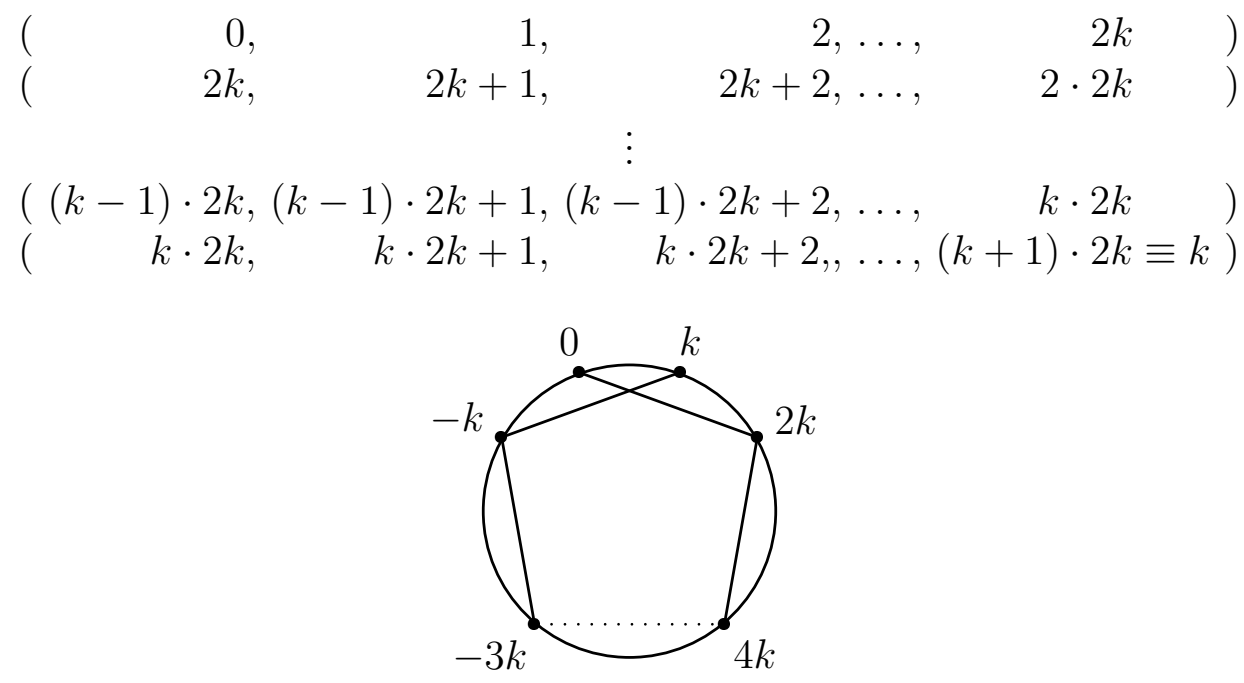

Figure 2: The relevant chords of Lemma 8 ,

By assumption, each of them must have a repeated color, so defining $c_{i}=\operatorname{col}(i \cdot k,(i+$ $2) \cdot k$ ) for $i \in \mathbb{Z} / m \mathbb{Z}$, we may conclude that

$$
\begin{aligned}
& c_{0}=\operatorname{col}(\quad 0,2 k) \in\{\quad 0, \quad 1, \ldots, 2 k-1\}, \\
& c_{2}=\operatorname{col}(2 k, 2 \cdot 2 k) \in\{\quad 2 k, \quad 2 k+1, \ldots, 2 \cdot 2 k-1\} \text {, } \\
& c_{2(k-1)}=\operatorname{col}((k-1) \cdot 2 k, k \cdot 2 k) \in\{(k-1) \cdot 2 k,(k-1) \cdot 2 k+1, \ldots, k \cdot 2 k-1\}, \\
& c_{-1}=c_{2 k}=\operatorname{col}(\quad k \cdot 2 k, \quad k) \in\{\quad k \cdot 2 k, \quad k \cdot 2 k+1, \ldots, \quad k-1\} \text {. }
\end{aligned}
$$

Now consider the $(2 k+1)$-cycle $(0,2 k, 4 k, \ldots,(k-1) \cdot 2 k, k \cdot 2 k, k, k-1, k-2, \ldots, 1)$; it has colors $\{0,1, \ldots, k-1\} \cup\left\{c_{0}, c_{2}, \ldots, c_{2 k}\right\}$. This collection must have a repeated color. But none of $c_{2}, \ldots, c_{2(k-1)}$ can contribute a repeated color, so we can conclude that one of $c_{0}$ and $c_{-1}=c_{2 k}$ is a member of $\{0,1, \ldots, k-1\}$. Notice, now, that we 
may translate this argument to also conclude that "either $c_{i}$ or $c_{i-1}$ is a member of $\{i \cdot k, i \cdot k+1, \ldots,(i+1) \cdot k-1\} "$.

By symmetry, assume that $c_{0} \in\{0,1, \ldots, k-1\}$. It follows that $c_{1} \in\{k, k+1, \ldots, 2 k-$ $1\}$ and, in general, $c_{i} \in\{i \cdot k, i \cdot k+1, \ldots,(i+1) \cdot k-1\}$. Now for the contradiction: consider the cycle $(0,2 k, \ldots, k \cdot 2 k, k, 3 k, \ldots, k \cdot(2 k-1))$, illustrated in Figure 3 . The colors along the edges are precisely the $c_{i}$, no two of which can be equal!

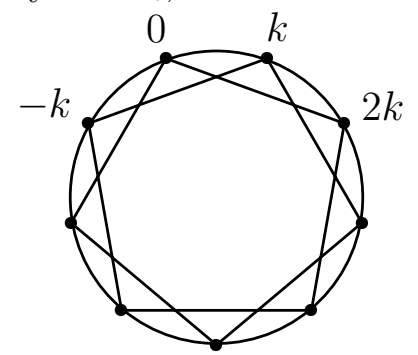

Figure 3: The eventual contradiction in the proof of Lemma 8 ,

Before proceeding further, we make some definitions and observations.

Definition. Let $\mathbb{N}(2)$ denote the set $\{2,3,4, \ldots\}$. With the operation $m \circ n=m+n-2$ of Lemma 1, $\mathbb{N}(2)$ becomes a monoid; moreover, by the map $n \mapsto n-2$, it is isomorphic to $\mathbb{N}$ under addition. This observation is useful because of its interaction with "absent" rainbow cycle lengths.

Thus, let the spectrum of a coloring col be the set of absent lengths of rainbow cycles, that is, $\{n \geq 2 \mid$ col does not contain a rainbow $n$-cycle $\}$. Notice that by definition, all spectra contain 2 .

Then by Lemma 1, the spectrum of a coloring is a submonoid of $\mathbb{N}(2)$. This will allow us to apply the well-known Claim 9. perhaps first published by Sylvester.

Claim 9 ("Frobenius coin-exchange problem" |Syl84|). If a submonoid of $\mathbb{N}$ under addition contains the relatively prime integers $a$ and $b$, then it contains all integers greater than or equal to $(a-1)(b-1)$. (In other words, every sufficiently large integer can be written as a nonnegative integer linear combination of $a$ and b.)

Equipped with these tools, the proof of the following lemma is immediate.

Lemma 10. If a coloring contains no rainbow $n$-cycles, where $n=2 k+1$, and no rainbow $m$-cycles, where $m=k \cdot(2 k+1)$, then it contains no rainbow $M$-cycles for all $M \geq 4 k^{3}-2 k^{2}-8 k+8=n^{3} / 2-2 n^{2}-3 n / 2+11$.

Proof. Note that since $k \cdot(2 k+1)-2 \equiv-1(\bmod (2 k+1)-2)$ (a fact we will use later as well), it follows that $(2 k+1)-2$ and $k \cdot(2 k+1)-2$ are relatively prime. Thus, by using Claim 9 and the isomorphism between $(\mathbb{N}(2), \circ)$ and $\mathbb{N}$ we obtain the desired bound $4 k^{3}-2 k^{2}-8 k+8$ after a basic computation. 
Clearly, Lemma 8 and Lemma 10 together imply the following Ramsey-type result, since $n^{3} / 2 \geq n^{3} / 2-2 n^{2}-3 n / 2+11$ for $n \geq 2$.

Theorem 11 (weaker version of Theorem 5). Suppose $n$ is an odd integer. If a coloring does not contain a rainbow $n$-cycle, it also does not contain a rainbow $m$-cycle for all sufficiently large $m$; in particular, a cubic bound $m \geq n^{3} / 2$ suffices.

\section{Proof of the Main Result}

We now prove the main result, which as in Section 5. will build on two intermediate lemmas.

Lemma 12. If a coloring does not contain a rainbow $n$-cycle, where $n=2 k+1>3$ is odd, it also does not contain a rainbow $m$-cycle, where $m=3 n-6=6 k-3$.

Remark. The $k=2$ case yields the result that the absence of rainbow 5-cycles implies the absence of rainbow 9-cycles.

Proof. The proof of this lemma has the same basic structure as that of Lemma 8 , but the cycles we consider are different. As before, we prove the contrapositive. Assume that we have a coloring col of $K_{m}$ such that there is a rainbow (Hamiltonian) $m$-cycle but no rainbow $n$-cycle. Without loss of generality, we may number the vertices of the $K_{m}$ by residues modulo $m$ and insist that $\operatorname{col}(i, i+1)=i$ for $i \in \mathbb{Z} / m \mathbb{Z}$. Note that in this proof, we will encounter more complicated $n$-cycles, but we will leave out the explicit verification of their length.

Consider the following three $n$-cycles: (see Figure 4 for a visual accompaniment - all of the chords of the cycles are either along drawn edges or along the arcs of the outside cycle)

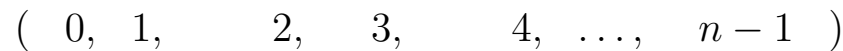

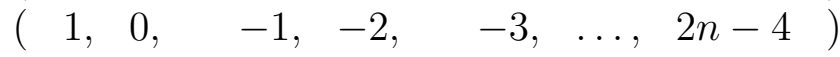

$$
\begin{aligned}
& (1, \quad 0, n-1, \quad n, n+1, \ldots, 2 n-4)
\end{aligned}
$$

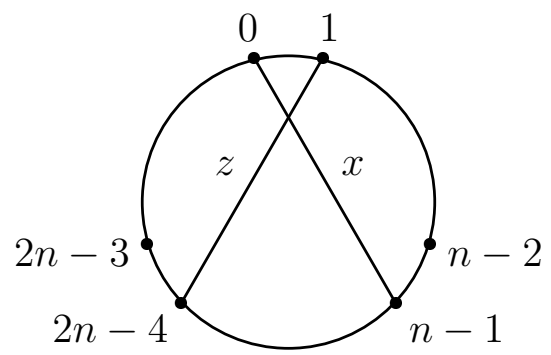

Figure 4: The chords in the first step of Lemma 12, as well as useful vertices to consider immediately after. 
Letting $x=\operatorname{col}(0, n-1)$ and $z=\operatorname{col}(1,2 n-4)$, the colors along the edges of these cycles are as follows:

$$
\begin{aligned}
& \left(\begin{array}{rrrrrr}
0, & 1, & 2, & 3, & 4, & \ldots,
\end{array}\right.
\end{aligned}
$$

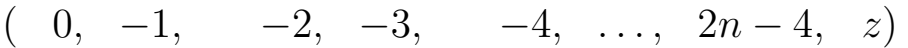

$$
\begin{aligned}
& (0, \quad x, n-1, \quad n, \quad n+1, \ldots, \quad 2 n-3, \quad z)
\end{aligned}
$$

Now assume there are no rainbow cycles. If $x$ is not 0 , it is between 1 and $n-2$; similarly, if $z$ is not 0 , it is between -1 and $-(n-2)$. It follows that if neither $x$ nor $z$ is 0 , the third cycle is a rainbow cycle, a contradiction. It follows that either $x$ or $z$ is 0 . Furthermore, let $y=\operatorname{col}(n-2,2 n-3)$; by symmetry, we can also apply this argument to $x$ and $y$ to deduce that either $x$ or $y$ is $n-2$, and similarly for $y$ or $z$ 's being $2 n-4$. Altogether, either $x=0, y=n-2$, and $z=2 n-4$ (call this "orientation + "); or $x=n-2, y=2 n-4$, and $z=0$ (call this "orientation -").

In a sense, our previous argument was "centered" on the edge $(0,1)$. By additive symmetry, we may also use the same argument centered at $(i, i+1)$ for any residue $i \in \mathbb{Z} / m \mathbb{Z}$; thus, we can assign an "orientation \pm " to every residue $i$ (by construction, though, the orientations of $i, i+m / 3$, and $i+2 m / 3$ are the same). Let $\Delta$ be a fixed integer; since $m$ is odd, there exists a residue $x$ such that $x$ and $x+\Delta$ have the same orientation. Without loss of generality, we may assume that $x=0$ and that the orientation is "orientation + ." In the following, we let $\frac{n-3}{2}$ be our particular choice of $\Delta$ (note that since $n>3, \Delta>0$ ). Figure 5 illustrates all of the edge colors that we may assume without any loss of generality.

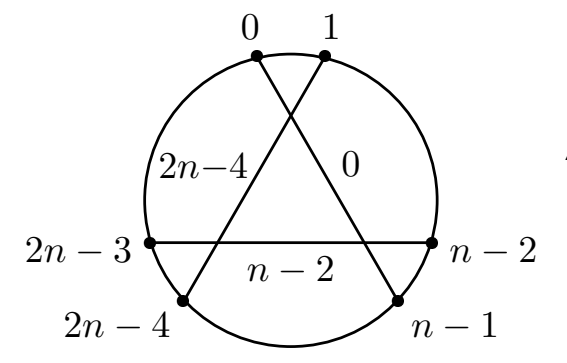

"Orientation +" centered at $(0,1)$

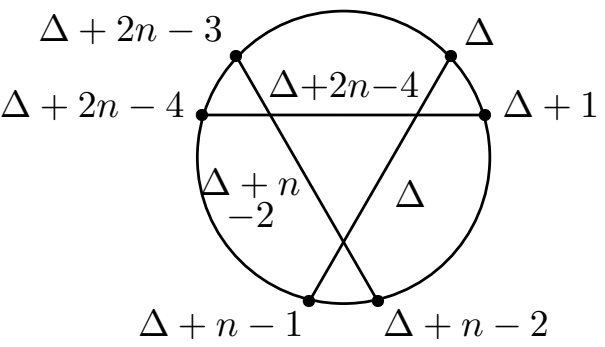

"Orientation +" centered at $(\Delta, \Delta+1)$ where $\Delta=\frac{n-3}{2}$

Figure 5: The edge colors we may assume without loss of generality in the next step of Lemma 12. They are illustrated on two separate diagrams for clarity.

Now let $t=\operatorname{col}(0, n-2)$; we will show that $t \in\{0, n-2\}$. Before continuing, let us define the shorthand notation $x \nearrow y$ to mean $x, x+1, x+2, \ldots, y$ (read: " $x$ up to $y$ ") and $x \searrow y$ to mean $x, x-1, x-2, \ldots, y$ (read: " $x$ down to $y$ "); Then in this notation, examine the following $n$-cycles and their corresponding edge colors, illustrated separately in Figure 6 : 
Respectively, the cycle and its edge colors
(a) $(0 \nearrow \Delta, \Delta+n-1 \searrow n-2)$
$(0 \nearrow \Delta, \Delta+n-2 \searrow n-2, t)$
(b) $(0 \nearrow \Delta+1, \Delta+2 n-4 \searrow 2 n-3, n-2)$
(c) $\begin{array}{r}(0, n-1 \nearrow \Delta+n-2, \\ \Delta+2 n-3\end{array}$
$(0 \nearrow \Delta, \Delta+2 n-4 \searrow 2 n-3, n-2, t)$$$
\Delta+2 n-3 \searrow 2 n-3, n-2)
$$$$
(0, n-1 \nearrow \Delta+n-2 \text {, }
$$$$
\Delta+2 n-2 \searrow 2 n-3, n-2, t)
$$
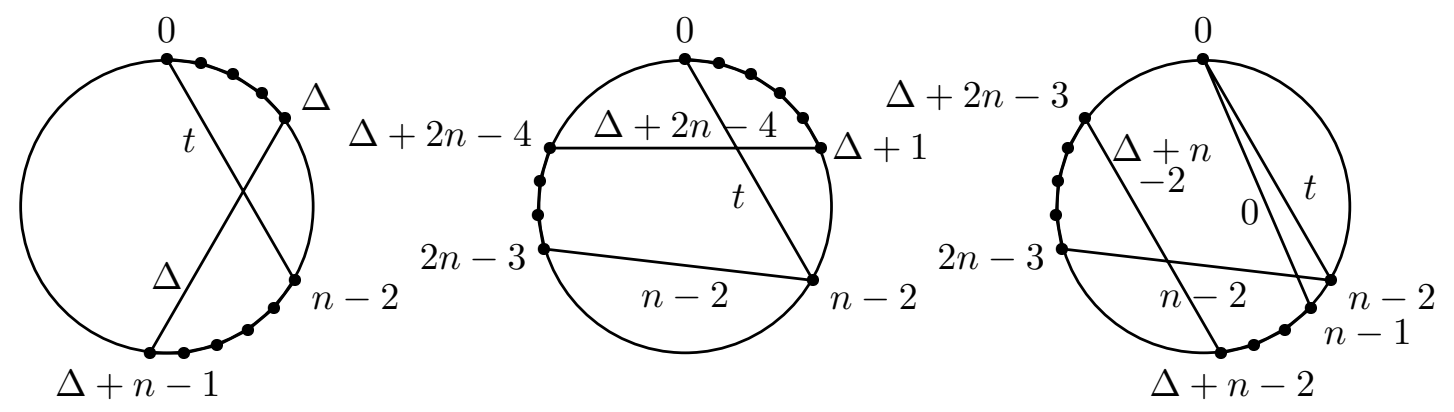
(a) $(0 \nearrow \Delta, \Delta+n-1 \searrow$ $n-2)$
(b) $(0 \nearrow \Delta+1, \Delta+2 n-4 \searrow$
(c) $(0, n-1 \nearrow \Delta+n-2$, $2 n-3, n-2)$ $\Delta+2 n-3 \searrow 2 n-3, n-2)$.

Figure 6: The three relevant $n$-cycles in the middle of the argument in Lemma 12.

Note that one of these cycles would be a rainbow cycles unless $t$ repeats a color found on all of them: either 0 or $n-2$. Thus, as desired, $t=\operatorname{col}(0, n-2) \in\{0, n-2\}$; by symmetry, it follows that $\operatorname{col}(n-2,2 n-4) \in\{n-2,2 n-4\}$ and $\operatorname{col}(2 n-4,0) \in\{2 n-4,0\}$. By enumerating the cases, one can check that there necessarily exists an $i$ such that $\operatorname{col}(i(n-2),(i+1)(n-2))+n-2=\operatorname{col}((i+1)(n-2),(i+2)(n-2))$. (See Figure 7 particularly subfigure (a), for continued visual accompaniment.) Our final contradiction will be to show that this is impossible; by symmetry, we may assume that this happens when $i=0$.

We can rule out the possibility $\operatorname{col}(0, n-2)=0$ and $\operatorname{col}(n-2,2 n-4)=n-2$ by considering the $n$-cycle $(n-2,2 n-4 \nearrow 0)$ which would then have colors $(n-2,2 n-4 \nearrow 0)$, a contradiction since no colors repeat and thus this cycle is rainbow. We can rule out the only other possibility $\operatorname{col}(0, n-2)=n-2$ and $\operatorname{col}(n-2,2 n-4)=2 n-4$ by considering the $n$-cycle $(0 \nearrow \Delta, \Delta+n-1 \nearrow 2 n-4, n-2)$ which would then have colors $(0 \nearrow \Delta, \Delta+n-1 \nearrow 2 n-4, n-2)$, also a contradiction. We have thus obtained a contradiction in all cases!

Before we can complete the proof of the main result, we make a preparatory claim regarding monoids; however, we need a stronger version than before. The analog of Claim 9 for three variables is well-studied (see, for example, [BS62, Vit75, Dav94]), and many special cases have been solved and algorithms have been developed. However, for completeness, we use a self-contained ad-hoc argument applicable in our particular situation. 


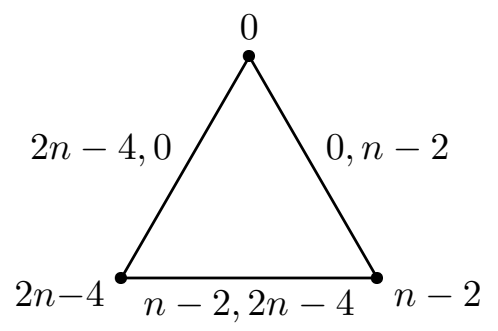
joining $0, n-2$, and $2 n-4$.

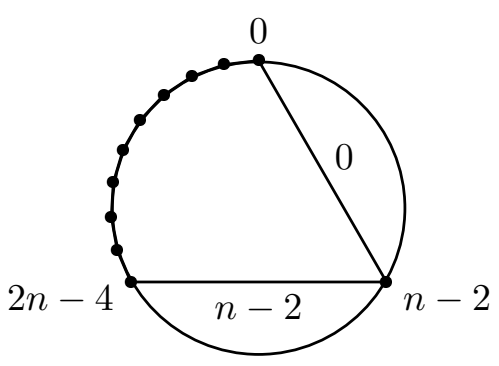

(b) Ruling out $\operatorname{col}(0, n-2)=$ 0 and $\operatorname{col}(n-2,2 n-4)=n-$ 2 .

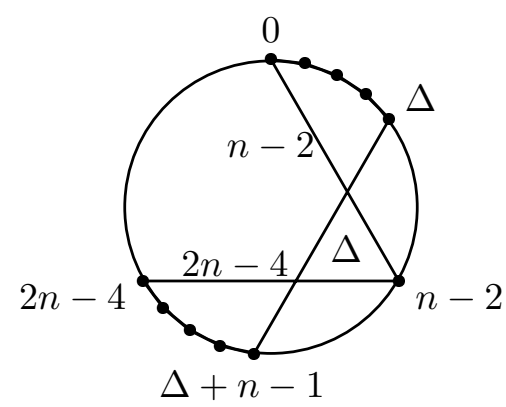

(c) Ruling out $\operatorname{col}(0, n-2)=$ $n-2$ and $\operatorname{col}(n-2,2 n-4)=$ $2 n-4$.

Figure 7: The eventual contradiction in Lemma 12.

Claim 13. Suppose a submonoid of $\mathbb{N}$ under addition contains the integers $a<b<c$, with $a \geq 3$ odd, $b \equiv-2(\bmod a)$, and $c \equiv-1(\bmod a)$ (thus, $a$ and $b$ are relatively prime, as are $a$ and $c)$. Then the submonoid contains all integers greater than or equal to $a b / 2-a-3 b / 2+c+1$.

Proof. It suffices to prove the claim for the submonoid generated by $a, b$, and $c$.

For each residue $r$ modulo $a$, consider the smallest number in the submonoid congruent to $r$ (modulo $a$ ). The smallest number in this submonoid that is congruent to $r \equiv$ $-(2 x) \bmod a$, where $0 \leq 2 x<a$, is $b x$; similarly, the smallest number congruent to $r \equiv-(2 x+1) \bmod a$, where $0<2 x+1<a$, is $b x+c$. The largest of these numbers, ranging over all residues $r$ modulo $a$, occurs for $r \equiv 2 \equiv-(a-2) \equiv-\left(2 \frac{a-3}{2}+1\right) \bmod a$, in which case it is $y=b \frac{a-3}{2}+c$. By choice of $y$, all numbers greater than $y-a$ are in the monoid, as desired.

This enables us to prove the following lemma and establish the main result.

Lemma 14. If a coloring contains no rainbow $n$-cycles, where $n=2 k+1>3$, and no rainbow $m_{1}$-cycles and $m_{2}$-cycles, where $m_{1}=k \cdot(2 k+1)$ and $m_{2}=6 k-3$, then it contains no rainbow $M$-cycles for all $M \geq 8 k^{2}-8 k+12=2 n^{2}-13 n+23$.

Proof. Since $n-2$ and $m_{2}-2$ are relatively prime, one approach is to simply proceed as in the proof of Lemma 10 and consider only $n$ and $m_{2}$. This approach, using Claim 9 . yields a bound of $12 k^{2}-24 k+14=3 n^{2}-18 n+29$, which is approximately a constant multiplicative factor worse than the desired bound.

We shall use both $m_{1}$ and $m_{2}$ to derive a better result. Apply Claim 13 above with $a=n-2, b=m_{2}-2$, and $c=m_{1}-2$, and use the isomorphism between $\mathbb{N}$ and $\mathbb{N}(2)$. One obtains the desired bound of $8 k^{2}-8 k+12=2 n^{2}-13 n+23$.

As before, Lemma 12 and Lemma 14 together complete the proof of Theorem 5 since $2 n^{2} \geq 2 n^{2}-13 n+23$ for $n \geq 2$. 
Theorem 5. Suppose $n$ is an odd integer. If a coloring does not contain a rainbow ncycle, it also does not contain a rainbow $m$-cycle for all sufficiently large $m$; in particular, $m \geq 2 n^{2}$ suffices.

\section{Further Directions}

This paper leaves open a few avenues of further experimentation which interest the author. We state some of these problems.

Problem 1. Completely characterize when the existence of a rainbow $m$-cycle implies the existence of a rainbow $n$-cycle.

Problem 2. Let $g(n)$ be the smallest value of $M$ such that if a coloring does not contain a rainbow $n$-cycle, where $n$ is odd, then it also does not contain a rainbow $m$-cycle for all $m \geq M$. Determine $g(n)$ for specific cases or in general.

Remark. For example, computer experimentation (see Appendix $\mathbb{\text { A }}$ ) yields $g(5)=8$, $g(7)=11, g(9)=15$, and $15 \leq g(11) \leq 34$.

Problem 3. In general, Theorem 5 shows that $g(n)$ is at most quadratic in $n$. Is $g(n)$ actually subquadratic?

Finally, we note that it is possible to make progress in this area. An earlier version of this paper made the following conjecture based on computer evidence; it was established in a recent paper of Vojtěchovský Voj06.

Conjecture. The asymptotic behavior of a spectrum $S$ can be classified into three categories: either (a) $S$ contains all sufficiently large numbers, (b) $S$ contains all sufficiently large even numbers, or (c) $S$ contains all sufficiently large numbers congruent to 2 mod 4 . In terms of monoids, the spectrum becomes regular either modulo one, two, or four.

\section{Acknowledgments}

The author wishes to thank Professor Daniel Kleitman of the Massachusetts Institute of Technology for guiding this work, as well as Jacob Fox, Matt Ince, Petr Vojtěchovský, and anonymous referees for helpful comments.

\section{References}

[Alo83] N. Alon, On a conjecture of Erdös, Simonovits, and Sós concerning antiRamsey theorems, J. Graph Theory 7 (1983), no. 1, 91-94.

[BEGS89] S. A. Burr, P. Erdős, R. L. Graham, and V. T. Sós, Maximal anti-Ramsey graphs and the strong chromatic number, J. Graph Theory 13 (1989), no. 3, 263-282. 
[BES $\left.{ }^{+} 91\right] \quad$ S. A. Burr, P. Erdös, V. T. Sós, P. Frankl, and R. L. Graham, Further results on maximal anti-Ramsey graphs, Graph theory, combinatorics, and applications, Vol. 1 (Kalamazoo, MI, 1988), Wiley-Intersci. Publ., Wiley, New York, 1991, pp. 193-206.

[BPV] R. N. Ball, A. Pultr, and P. Vojtěchovský, Colored graphs without colorful cycles, Combinatorica, to appear.

[BS62] A. Brauer and J. E. Shockley, On a problem of Frobenius, J. Reine Angew. Math. 211 (1962), 215-220.

[Dav94] J. L. Davison, On the linear Diophantine problem of Frobenius, J. Number Theory 48 (1994), no. 3, 353-363.

[ESS75] P. Erdős, M. Simonovits, and V. T. Sós, Anti-Ramsey theorems, Infinite and finite sets (Colloq., Keszthely, 1973; dedicated to P. Erdős on his 60th birthday), Vol. II, North-Holland, Amsterdam, 1975, pp. 633-643. Colloq. Math. Soc. János Bolyai, Vol. 10.

[JW03] T. Jiang and D. B. West, On the Erdös-Simonovits-Sós conjecture about the anti-Ramsey number of a cycle, Combin. Probab. Comput. 12 (2003), no. 5-6, 585-598, Special issue on Ramsey theory.

[MBNL05] J. J. Montellano-Ballesteros and V. Neumann-Lara, An anti-Ramsey theorem on cycles, Graphs Combin. 21 (2005), no. 3, 343-354.

[Syl84] J. J. Sylvester, Question 7382, Mathematical Questions from the Educational Times 41 (1884), 21.

[Vit75] Y. Vitek, Bounds for a linear diophantine problem of Frobenius, J. London Math. Soc. (2) 10 (1975), 79-85.

[Voj06] P. Vojtěchovský, Missing lengths of rainbow cycles, Preprint, Aug 2006.

\section{A Computer Results}

An original $\mathrm{C}++$ program was used to exhaustively determine (by searching through the space of all possible colorings by always coloring the most-constrained edge), for small $n$ and $m$, whether or not it is possible to color $K_{m}$ so that there exists a rainbow $m$-cycle but no rainbow $n$-cycle. These results are summarized in Table 1. Some of these results have been confirmed by Petr Vojtěchovský; in particular, the case $m=n+1>3$ is known (this corresponds to the top entry of each column). [BPV] 


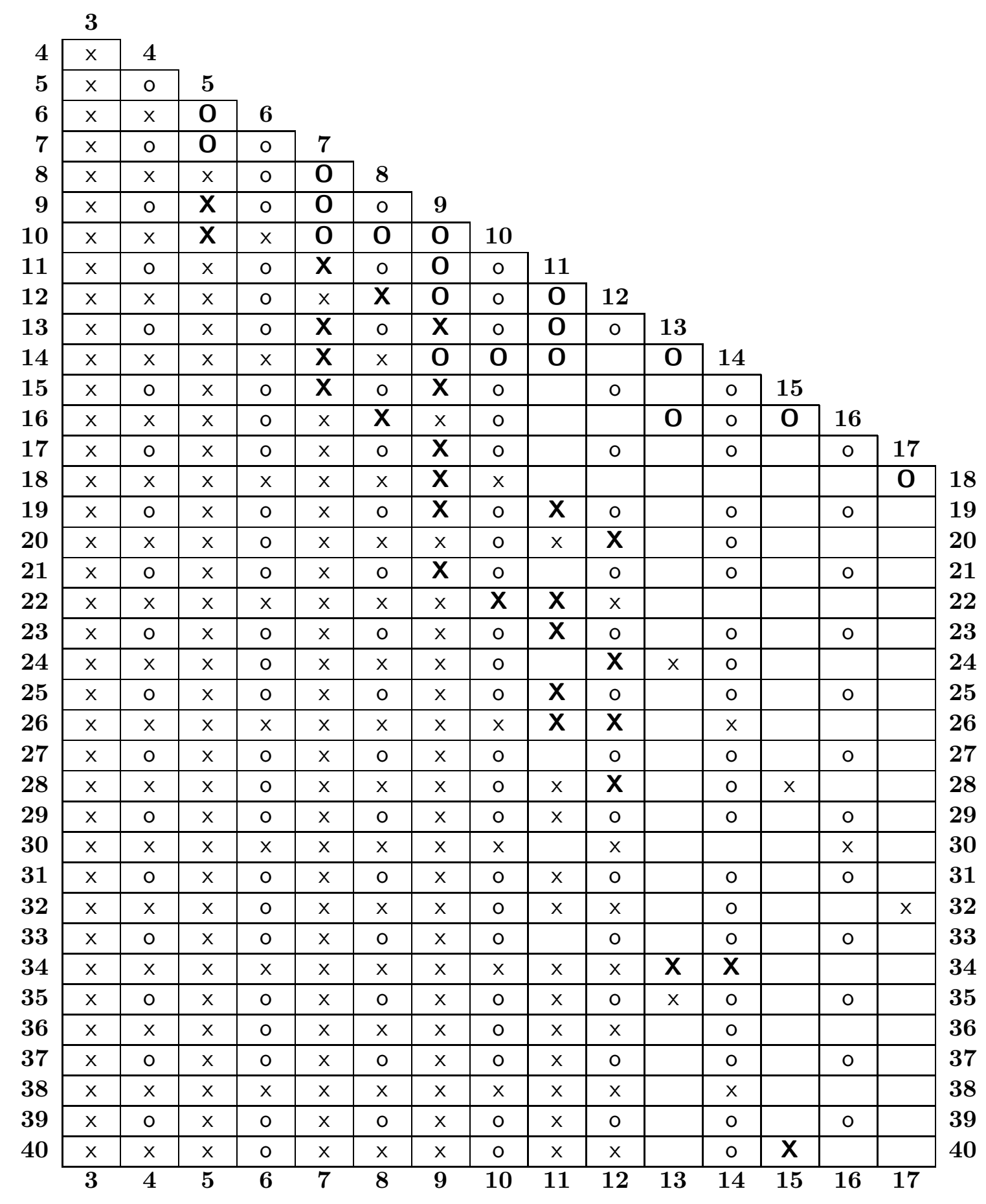

Table 1: Data obtained from a computer program. For example, the lowest ' $\mathbf{X}$ ' in column 5 means that the absence of rainbow 5 -cycles implies the absence of rainbow 10-cycles. The symbols mean the following: 'o' means that Claim [6] or Claim 7 implies that the existence of the larger length is possible, while ' $\mathbf{O}$ ' means that these Claims do not apply but a specific example was found. ' $\mathbf{X}$ ' means that the program determined that the larger length could not occur, while ' $x$ ' are consequences of Lemma 1. An empty square means no results were obtained. 